\title{
Contamination of the Accuracy of the Combined-Field Integral Equation With the Discretization Error of the Magnetic-Field Integral Equation
}

\author{
Levent Gürel, Fellow, IEEE, and Özgür Ergül, Student Member, IEEE
}

\begin{abstract}
We investigate the accuracy of the combined-field integral equation (CFIE) discretized with the Rao-Wilton-Glisson (RWG) basis functions for the solution of scattering and radiation problems involving three-dimensional conducting objects. Such a low-order discretization with the RWG functions renders the two components of CFIE, i.e., the electric-field integral equation (EFIE) and the magnetic-field integral equation (MFIE), incompatible, mainly because of the excessive discretization error of MFIE. Solutions obtained with CFIE are contaminated with the MFIE inaccuracy, and CFIE is also incompatible with EFIE and MFIE. We show that, in an iterative solution, the minimization of the residual error for CFIE involves a breakpoint, where a further reduction of the residual error does not improve the solution in terms of compatibility with EFIE, which provides a more accurate reference solution. This breakpoint corresponds to the last useful iteration, where the accuracy of CFIE is saturated and a further reduction of the residual error is practically unnecessary.
\end{abstract}

Index Terms-Accuracy analysis, combined-field integral equation (CFIE), iterative methods, surface integral equations.

\section{INTRODUCTION}

$\mathbf{S}$ URFACE integral equations are commonly used to formulate scattering and radiation problems in electromagnetics [1]. When a problem involves closed conducting surfaces, it can be formulated with the electric-field integral equation (EFIE), the magnetic-field integral equation (MFIE), or the combined-field integral equation (CFIE), which is the convex combination of EFIE and MFIE. In general, CFIE is preferred over EFIE and MFIE, because CFIE is free of the internal-resonance problem [2], and its discretization produces well-conditioned matrix equations, which are easy to solve iteratively [3]-[5] with accelerated methods, such as the fast multipole method (FMM) [6] and the multilevel fast multipole algorithm (MLFMA) [7].

Manuscript received January 09, 2009. First published June 05, 2009; current version published September 02, 2009. This work was supported by the Turkish Academy of Sciences in the framework of the Young Scientist Award Program (LG/TUBA-GEBIP/2002-1-12), by the Scientific and Technical Research Council of Turkey (TUBITAK) under Research Grants 105E172 and 107E136, and by contracts from ASELSAN and SSM.

The authors are with the Department of Electrical and Electronics Engineering and Computational Electromagnetics Research Center (BiLCEM), Bilkent University, TR-06800, Bilkent, Ankara, Turkey (e-mail: lgurel@ee.bilkent.edu.tr; ergul@ee.bilkent.edu.tr).

Color versions of one or more of the figures in this paper are available online at http://ieeexplore.ieee.org.

Digital Object Identifier 10.1109/TAP.2009.2024529
For numerical solutions of scattering and radiation problems involving three-dimensional objects with arbitrary shapes, it is common to apply a triangulation on the surface of the object and discretize integral equations by using a set of Rao-WiltonGlisson (RWG) functions defined on triangles [8]. In many applications, discretizations of EFIE with $\lambda / 10$ mesh size, where $\lambda$ is the wavelength, produce accurate results. Unfortunately, recent studies show that the same accuracy cannot be obtained with MFIE [9]-[19]. Induced currents and scattered or radiated electromagnetic fields calculated with MFIE can be significantly inaccurate, compared to EFIE, even when the same discretization is used for the two formulations. Investigations also show that the inaccuracy of MFIE is mainly due to insufficient discretization of the identity operator with the RWG functions [11], [19]-[21]. Even though the discretization of the identity operator is trivial, this operator behaves like an integro-differential operator with a strongly-singular kernel [11], [19], [20]. Due to the excessive discretization error of the identity operator in MFIE, matrix equations obtained with EFIE and MFIE are incompatible, i.e., a solution vector calculated by using MFIE does not satisfy EFIE with the desired level of accuracy, and vice versa. Being a convex combination of EFIE and MFIE, CFIE solutions are contaminated with the MFIE inaccuracy [17]. Therefore, coefficients obtained with CFIE are also incompatible with EFIE and MFIE systems.

In this study, we investigate the accuracy of CFIE discretized with the RWG functions. We show that, in an iterative solution, the minimization of the residual error of CFIE involves a breakpoint, where further reduction of the residual error does not improve the compatibility of the CFIE solution with EFIE and MFIE systems. Since EFIE produces more accurate results than CFIE, the breakpoint also corresponds to the last "useful" iteration, where the accuracy of the CFIE solution is saturated and cannot be improved any more. Then, a further reduction of the residual error is practically unnecessary. Our observations are demonstrated on three different electromagnetics problems depicted in Fig. 1. The radiation problem in Fig. 1(a) involves a $\lambda \times \lambda \times \lambda$ conducting box located at the origin. The box is excited with a Hertzian dipole located inside the box at $x=0.1 \lambda$. In the scattering problem depicted in Fig. 1(b), a $\lambda \times \lambda \times \lambda$ conducting box is illuminated by a plane wave propagating in the $-x$ direction with the electric field polarized in the $y$ direction. Finally, the scattering problem in Fig. 1(c) involves a conducting sphere with a radius of $1.5 \lambda$. Similar to the second problem, the sphere is illuminated by a plane wave propagating in the $-x$ direction with the electric field polarized in the $y$ di- 


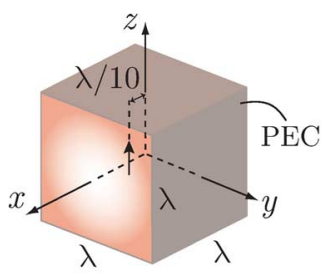

(a)

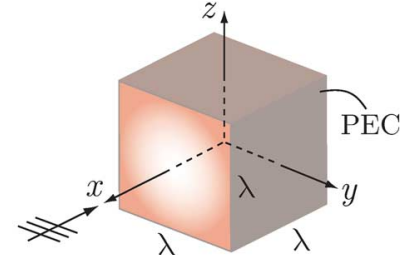

(b)

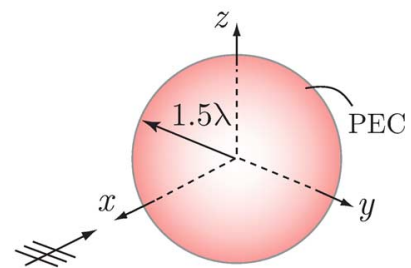

(c)

Fig. 1. Electromagnetics problems involving canonical objects. (a) A radiation problem involving a $\lambda \times \lambda \times \lambda$ conducting box excited with a Hertzian dipole located inside the box at $x=0.1 \lambda$. (b) A scattering problem involving a $\lambda \times$ $\lambda \times \lambda$ conducting box illuminated by a plane wave. (c) A scattering problem involving a sphere with a radius of $1.5 \lambda$ illuminated by a plane wave.

rection. Discretizations of the problems with the RWG functions on $\lambda / 10$ triangles lead to $2052 \times 2052$ (box) and $8364 \times 8364$ (sphere) matrix equations. Matrix elements are calculated with a maximum of $1 \%$ error by using singularity extraction techniques [22]-[25], Gaussian quadratures [26], and adaptive integration methods [27]. Iterative solutions of the matrix equations are performed by using the biconjugate-gradient-stabilized (BiCGStab) algorithm [28].

The rest of the paper is organized as follows. In Section II, we discuss the incompatibility of the integral-equation formulations, i.e., EFIE, MFIE, and CFIE. In Section III, we consider iterative solutions of CFIE and demonstrate the incompatibility of CFIE solutions with EFIE and MFIE systems in terms of residual errors. Then, Section IV investigates convergence to minimum achievable error in iterative solutions of EFIE and CFIE. For CFIE solutions, we demonstrate the relation between the last useful iteration to obtain the highest possible accuracy and the breakpoint for the compatibility with EFIE. Finally, Section V includes our concluding remarks.

\section{INCOMPATIBILITY OF INTEGRAL EQUATIONS}

For the solution of a scattering or radiation problem involving a closed conducting object, discretizations of EFIE and MFIE lead to $N \times N$ dense matrix equations in the form of

$$
\overline{\boldsymbol{Z}}_{E, M} \cdot \boldsymbol{x}=\boldsymbol{v}_{E, M}
$$

where $\overline{\boldsymbol{Z}}_{E, M}$ represents impedance matrices, $\boldsymbol{v}_{E, M}$ represents excitation vectors, and $\boldsymbol{x}$ represents the unknown coefficient vector. Due to the excessive discretization error in MFIE compared to EFIE, matrix equations obtained from the two formulations are incompatible, and their solutions are unequal, i.e.,

$$
\left(\overline{\boldsymbol{Z}}_{E}\right)^{-1} \cdot \boldsymbol{v}_{E}=\boldsymbol{x}_{E} \neq \boldsymbol{x}_{M}=\left(\overline{\boldsymbol{Z}}_{M}\right)^{-1} \cdot \boldsymbol{v}_{M}
$$

even when EFIE and MFIE solutions are not contaminated with internal resonances. In common implementations, where discretizations are performed by employing the RWG functions on $\lambda / 10$ triangulations, coefficients obtained with EFIE and MFIE may differ significantly [9]-[19]. Increasing the accuracy of implementations, especially for MFIE, by using finer triangulations and/or using higher-order basis functions reduces the discrepancy between EFIE and MFIE [10], [16]-[18].

In this paper, we investigate the accuracy of the conventional implementations, where the integral equations are discretized by employing the RWG functions on $\lambda / 10$ triangulations. Then, EFIE solutions are considered to be reference solutions, and we write

$$
\boldsymbol{x}_{M}=\boldsymbol{x}_{E}+\Delta \boldsymbol{x}_{M E}
$$

where the discrepancy between the coefficients obtained with EFIE and MFIE is interpreted as the error in MFIE. CFIE is obtained by combining EFIE and MFIE as

$$
\bar{Z}_{C} \cdot x_{C}=v_{C}
$$

where

$$
\begin{aligned}
\overline{\boldsymbol{Z}}_{C} & =\alpha \overline{\boldsymbol{Z}}_{E}+(1-\alpha) \overline{\boldsymbol{Z}}_{M} \\
\boldsymbol{v}_{C} & =\alpha \boldsymbol{v}_{E}+(1-\alpha) \boldsymbol{v}_{M}
\end{aligned}
$$

and $0 \leq \alpha \leq 1$. The coefficient vector obtained with CFIE is related to those obtained with EFIE and MFIE as

$$
\boldsymbol{x}_{C}=\alpha\left(\overline{\boldsymbol{Z}}_{C}\right)^{-1} \cdot \overline{\boldsymbol{Z}}_{E} \cdot \boldsymbol{x}_{E}+(1-\alpha)\left(\overline{\boldsymbol{Z}}_{C}\right)^{-1} \cdot \overline{\boldsymbol{Z}}_{M} \cdot \boldsymbol{x}_{M} .
$$

Using (3) and (5) in (7), we arrive at

$$
\boldsymbol{x}_{C}=\boldsymbol{x}_{E}+\Delta \boldsymbol{x}_{C E}
$$

where

$$
\Delta \boldsymbol{x}_{C E}=(1-\alpha)\left(\overline{\boldsymbol{Z}}_{C}\right)^{-1} \cdot \overline{\boldsymbol{Z}}_{M} \cdot \Delta \boldsymbol{x}_{M E}
$$

which can be interpreted as the CFIE solution being contaminated with the inaccuracy of MFIE.

It should be emphasized that this paper is not concerned with errors resulting from the internal resonances of EFIE and MFIE; all references to error and accuracy are related to discretization errors of the integral equations. It is well known that CFIE eliminates the respective null spaces of EFIE and MFIE, and hence is immune to the internal-resonance problem. At a specific frequency, the EFIE or MFIE solution may be corrupted with an internal-resonance error. However, even though CFIE is a combination of EFIE and MFIE, if a CFIE solution is obtained at the same frequency, it will not contain any internal-resonance error propagating from EFIE or MFIE. At that same frequency, both EFIE and MFIE solutions contain errors due to the discretizations of the integral equations. Such discretization errors are relatively small compared to the larger internal-resonance errors, and they may not be noticeable in the presence of that larger error. Nevertheless, they exist and contaminate the CFIE solution. It is interesting to note that a larger internal-resonance 


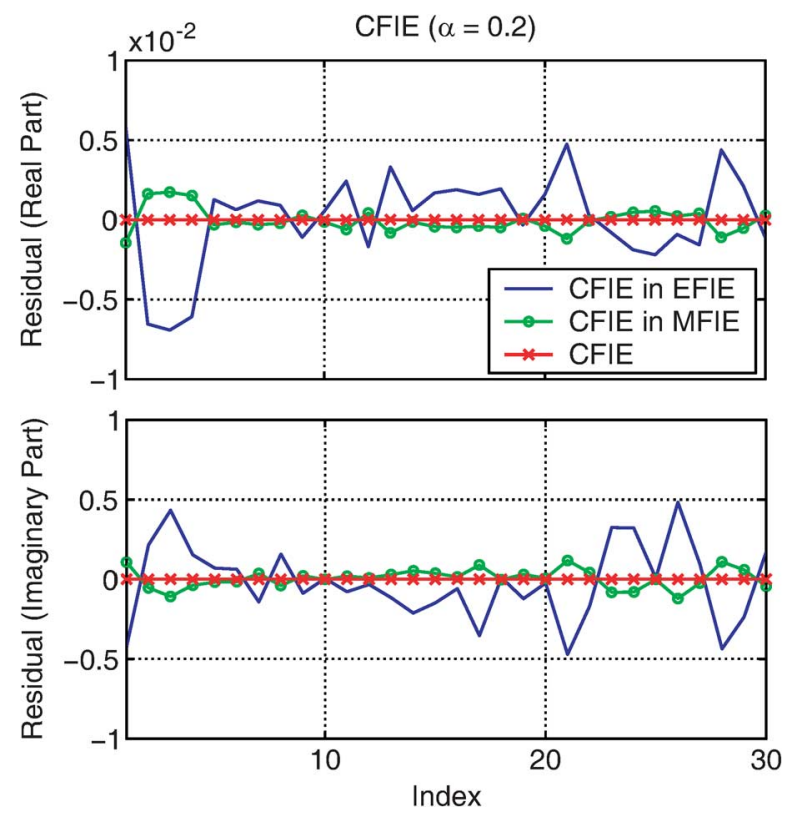

Fig. 2. First 30 elements of residual vectors $\boldsymbol{r}_{C}$ (CFIE), $\boldsymbol{r}_{C \rightarrow E}$ (CFIE in EFIE), and $\boldsymbol{r}_{C \rightarrow M}$ (CFIE in MFIE) for the iterative CFIE $(\alpha=0.2)$ solution of the radiation problem in Fig. 1(a). Residual vectors $\boldsymbol{r}_{C \rightarrow E}$ and $\boldsymbol{r}_{C \rightarrow M}$ are obtained by testing the coefficient vector $\boldsymbol{x}_{C}$ in matrix equations obtained with EFIE and MFIE, respectively.

error of MFIE does not propagate to the CFIE solution, but a smaller discretization error does.

\section{MINIMIZATION OF RESIDUAL ERROR IN ITERATIVE SOLUTIONS OF CFIE}

When the solution of CFIE is obtained by using an iterative algorithm, the residual vector

$$
\boldsymbol{r}_{C}=\boldsymbol{v}_{C}-\overline{\boldsymbol{Z}}_{C} \cdot \boldsymbol{x}_{C}
$$

is minimized. Using (5) and (6) in (10), the residual vector for CFIE can be written as

$$
\boldsymbol{r}_{C}=\alpha \boldsymbol{r}_{C \rightarrow E}+(1-\alpha) \boldsymbol{r}_{C \rightarrow M}
$$

where

$$
\begin{aligned}
\boldsymbol{r}_{C \rightarrow E} & =\boldsymbol{v}_{E}-\overline{\boldsymbol{Z}}_{E} \cdot \boldsymbol{x}_{C} \\
\boldsymbol{r}_{C \rightarrow M} & =\boldsymbol{v}_{M}-\overline{\boldsymbol{Z}}_{M} \cdot \boldsymbol{x}_{C}
\end{aligned}
$$

are residual vectors obtained by testing the CFIE solution in the EFIE and MFIE systems, respectively.

When the norm of $r_{C}$ in (11) is minimized in an iterative CFIE solution, norms of $\boldsymbol{r}_{C \rightarrow E}$ and $\boldsymbol{r}_{C \rightarrow M}$ are not necessarily minimized. As an example, we consider the solutions of the radiation and scattering problems in Fig. 1(a) and (b) involving a $\lambda \times \lambda \times \lambda$ conducting box, when the problems are formulated with CFIE. Iterations are stopped when the residual error

$$
\epsilon_{C}=\frac{\left\|\boldsymbol{r}_{C}\right\|_{2}}{\left\|\boldsymbol{v}_{C}\right\|_{2}}
$$

where $\|\cdot\|_{2}$ represents the $l^{2}$-norm of a vector, is reduced to below $10^{-6}$. Fig. 2 presents the first 30 elements of residual vectors $\boldsymbol{r}_{C}, \boldsymbol{r}_{C \rightarrow E}$, and $\boldsymbol{r}_{C \rightarrow M}$, denoted by "CFIE", "CFIE in

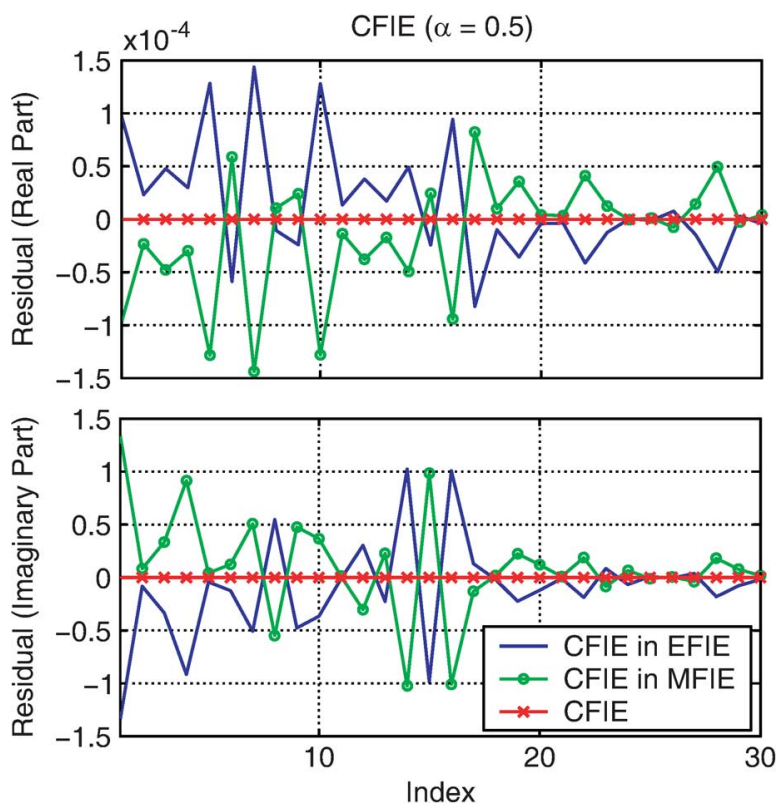

Fig. 3. First 30 elements of residual vectors $\boldsymbol{r}_{C}$ (CFIE), $\boldsymbol{r}_{C \rightarrow E}$ (CFIE in EFIE), and $\boldsymbol{r}_{C \rightarrow M}$ (CFIE in MFIE) for the iterative CFIE $(\alpha=0.5)$ solution of the scattering problem in Fig. 1(b). Residual vectors $\boldsymbol{r}_{C \rightarrow E}$ and $\boldsymbol{r}_{C \rightarrow M}$ are obtained by testing the coefficient vector $\boldsymbol{x}_{C}$ in matrix equations obtained with EFIE and MFIE, respectively.

EFIE", and "CFIE in MFIE", respectively, when $\alpha=0.2$ in the CFIE solution of the radiation problem in Fig. 1(a). We observe that $\boldsymbol{r}_{C \rightarrow E}$ and $\boldsymbol{r}_{C \rightarrow M}$ involve significantly larger elements than $\boldsymbol{r}_{C}$. Furthermore, the elements of $\boldsymbol{r}_{C \rightarrow E}$ are exactly four times those of $\boldsymbol{r}_{C \rightarrow M}$. This is because, as the norm of $\boldsymbol{r}_{C}$ is minimized, elements of $\boldsymbol{r}_{C \rightarrow E}$ and $\boldsymbol{r}_{C \rightarrow M}$ are scaled with respect to each other, i.e.,

$$
\boldsymbol{r}_{C \rightarrow E} \approx-\frac{(1-\alpha)}{\alpha} \boldsymbol{r}_{C \rightarrow M}
$$

instead of converging to zero. For $\alpha=0.2$, the ratio of

$$
-(1-\alpha) / \alpha=-4
$$

is manifested in Fig. 2. More precisely, the error criteria for the iterative solution, i.e.,

$$
\frac{\left\|\boldsymbol{r}_{C}\right\|_{2}}{\left\|\boldsymbol{v}_{C}\right\|_{2}}=\frac{\left\|\alpha \boldsymbol{r}_{C \rightarrow E}+(1-\alpha) \boldsymbol{r}_{C \rightarrow M}\right\|_{2}}{\left\|\boldsymbol{v}_{C}\right\|_{2}} \leq 10^{-6}
$$

is achieved without the minimization of $\left\|\boldsymbol{r}_{C \rightarrow E}\right\|_{2}$ and $\left\|\boldsymbol{r}_{C \rightarrow M}\right\|_{2}$.

Fig. 3 presents the first 30 elements of residual vectors $\boldsymbol{r}_{C}$, $\boldsymbol{r}_{C \rightarrow E}$, and $\boldsymbol{r}_{C \rightarrow M}$, when $\alpha=0.5$ in the CFIE solution of the scattering problem in Fig. 1(b). Similar to the previous example, $\boldsymbol{r}_{C \rightarrow E}$ and $\boldsymbol{r}_{C \rightarrow M}$, denoted by "CFIE in EFIE" and "CFIE in MFIE", respectively, involve significantly larger elements than $\boldsymbol{r}_{C}$, denoted by "CFIE." As opposed to the previous solution, however, $\boldsymbol{r}_{C \rightarrow E}=-\boldsymbol{r}_{C \rightarrow M}$ since

$$
-(1-\alpha) / \alpha=-1
$$



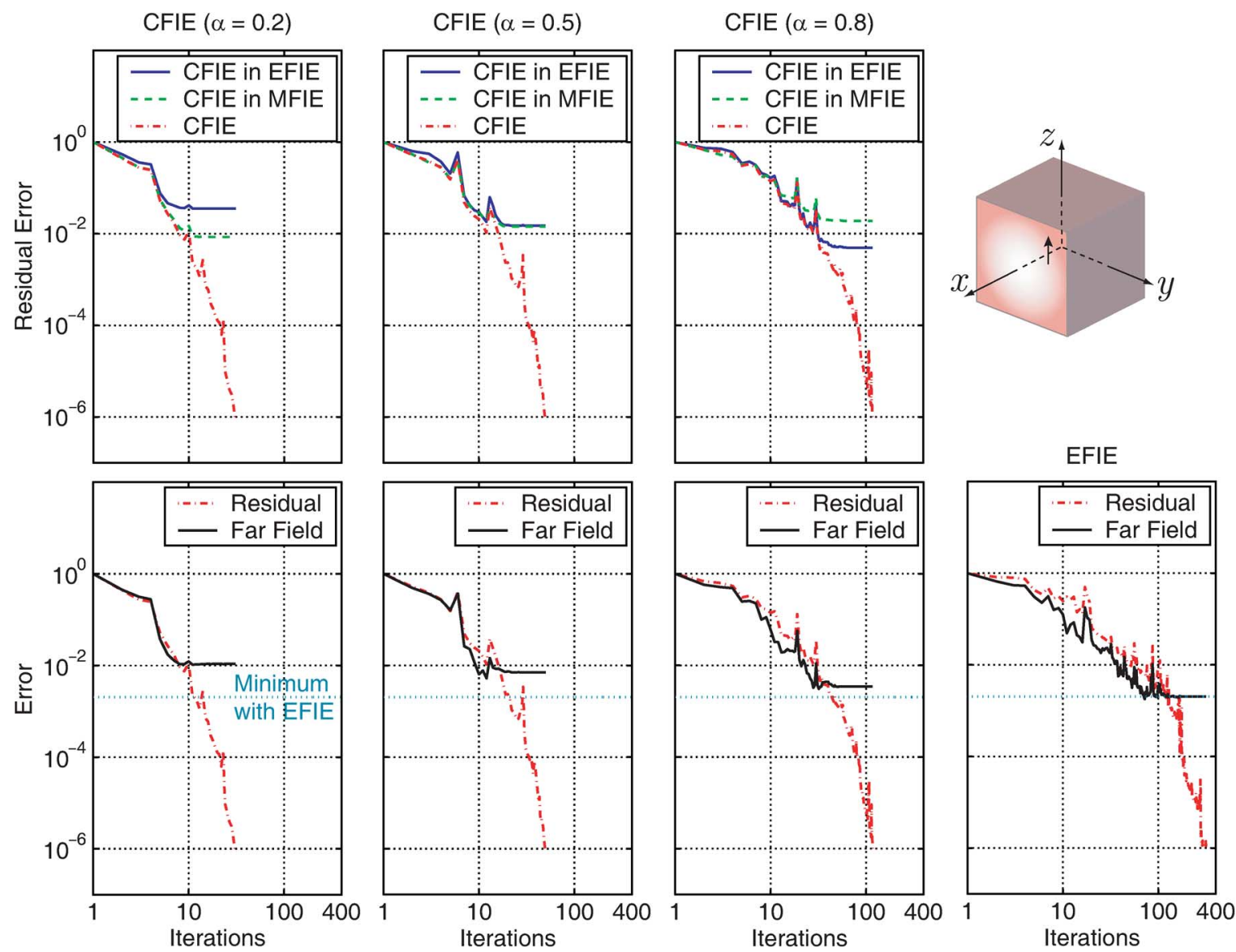

Fig. 4. Iterative solutions of the radiation problem in Fig. 1(a). Residual errors and the far-field error defined in (22) are plotted as a function BiCGStab iterations for EFIE and CFIE when $\alpha=0.2,0.5$, and 0.8 .

Depending on the value of $\alpha$, compatibility of the CFIE solution with the EFIE and MFIE systems varies, but the CFIE solution cannot satisfy both systems at the same time with the desired level of accuracy.

\section{CONVERgence to The Minimum ACHIEVABLE ERRoR}

In an iterative solution of a matrix equation obtained from the discretization of an integral-equation formulation, accuracy of the solution is saturated and the solution error cannot be reduced any further after a number of iterations. This is because there are various error sources, such as the numerical calculation of the matrix elements, and the overall error cannot be minimized by only reducing the residual error. Then, in an iterative solution, a further reduction of the residual error becomes meaningless after the minimum solution error is achieved.

As shown in Section III, a CFIE solution can be significantly incompatible with the EFIE and MFIE systems, even when the residual error is small. In fact, an iterative solution of CFIE involves a breakpoint, where a further reduction of the residual error does not improve the solution in terms of compatibility with EFIE and MFIE. Since EFIE is more accurate than CFIE, this breakpoint, where the compatibility of the CFIE solution with the EFIE system is maximized, corresponds to the last useful iteration to obtain the highest possible accuracy with CFIE. We note that the minimum achievable error in an EFIE solution depends on various error sources, and not only on the reduction of the residual error. In the case of CFIE, however, the excessive discretization error in the MFIE part becomes a major error source, and the minimum achievable error is closely related to the compatibility of the CFIE solution with the EFIE system.

Fig. 4 presents the iterative solutions of the radiation problem in Fig. 1(a) formulated with EFIE and CFIE. For CFIE, we consider three different combinations of EFIE and MFIE, i.e., $\alpha=0.2,0.5$, and 0.8. In Fig. 4, residual errors

$$
\epsilon_{E, C}=\frac{\left\|\boldsymbol{r}_{E, C}\right\|_{2}}{\left\|\boldsymbol{v}_{E, C}\right\|_{2}}
$$

are plotted with respect to BiCGStab iterations. For the CFIE solutions, we also plot

$$
\epsilon_{C \rightarrow E}=\frac{\left\|\boldsymbol{r}_{C \rightarrow E}\right\|_{2}}{\left\|\boldsymbol{v}_{E}\right\|_{2}} \text { and } \epsilon_{C \rightarrow M}=\frac{\left\|\boldsymbol{r}_{C \rightarrow M}\right\|_{2}}{\left\|\boldsymbol{v}_{M}\right\|_{2}}
$$

denoted by "CFIE in EFIE" and "CFIE in MFIE", respectively, in the first row of Fig. 4. Our observations are as follows.

- In the EFIE solution, the number of iterations to reduce the residual error $\epsilon_{E}$ to below $10^{-6}$ is 316 .

- In the CFIE solutions, the number of iterations is 31,50 , and 117 when $\alpha=0.2,0.5$, and 0.8 , respectively.

- Compatibility of the CFIE solution with the EFIE and MFIE systems is saturated at about the 10th, 20th, and 50th iterations, respectively. The values of $\epsilon_{C \rightarrow E}$ and $\epsilon_{C \rightarrow M}$, 

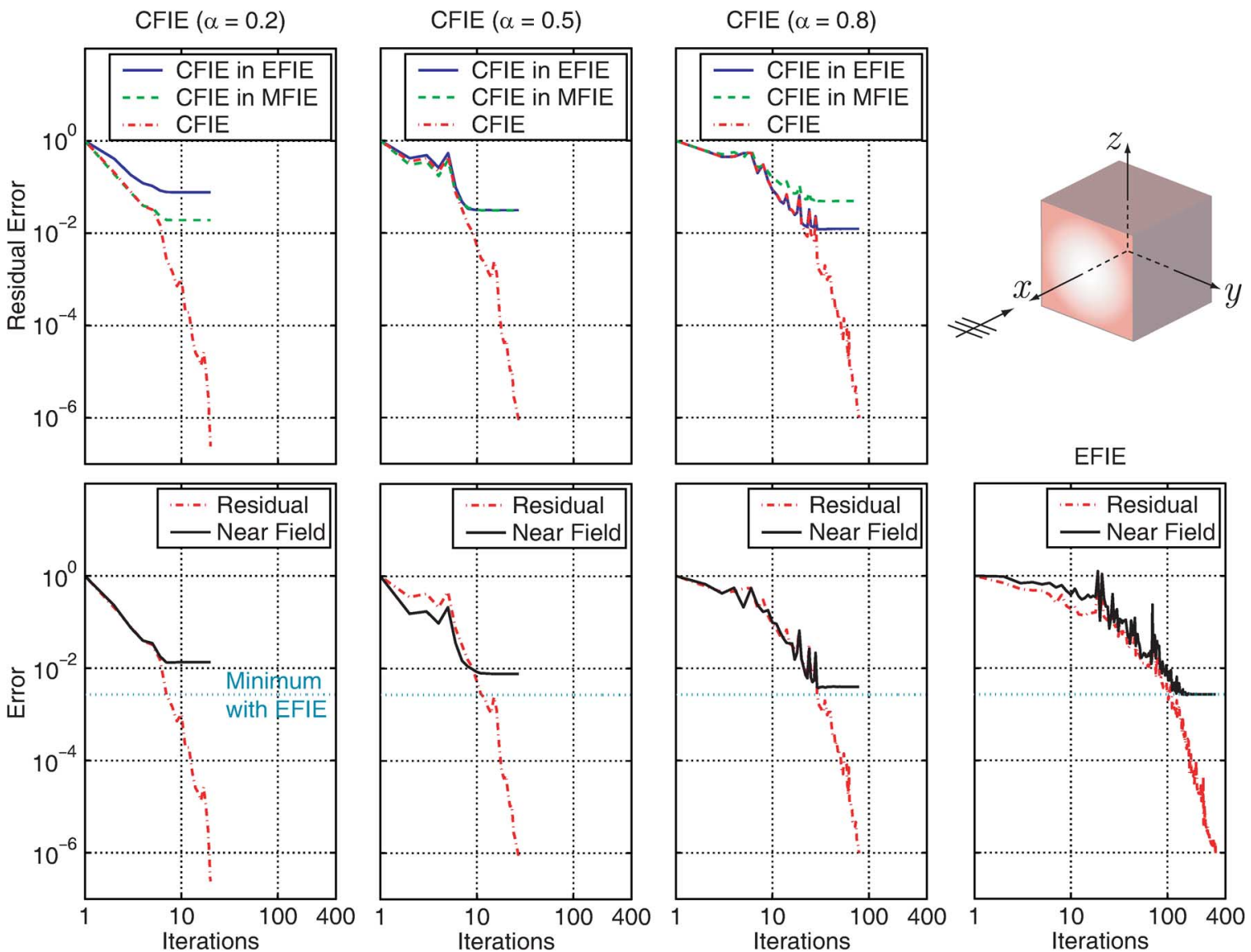

Fig. 5. Iterative solutions of the scattering problem in Fig. 1(b). Residual errors and the near-field error defined in (24) are plotted as a function BiCGStab iterations for EFIE and CFIE when $\alpha=0.2,0.5$, and 0.8 .

which are obtained by testing the coefficient vector in the EFIE and MFIE systems, respectively, become steady after these breakpoints, and they do not drop further as iterations continue.

- The maximum compatibility of the CFIE solution with the EFIE or MFIE system depends on the value of $\alpha$. This is in agreement with the results in Section III.

For the radiation problem in Fig. 1(a), we also calculate the error in the far-zone electric field at each iteration in addition to the residual error. We compute the total electric field in the far zone on the $x-y$ plane as

$$
E_{\text {tot }}^{\infty}[p]=\lim _{R \rightarrow \infty} R\left\{\left|E_{\text {tot }}^{\theta}\left(R, \theta, \phi_{p}\right)\right|^{2}+\left|E_{\text {tot }}^{\phi}\left(R, \theta, \phi_{p}\right)\right|^{2}\right\}^{1 / 2}
$$

where $E_{\text {tot }}^{\theta}$ and $E_{\text {tot }}^{\phi}$ are the $\theta$ and $\phi$ components of the total electric field, $\theta=\pi / 2$, and $\phi_{p}=(p-1) \pi / 180$ for $p=$ $1,2, \ldots, 360$. The total electric field is obtained by combining the incident field radiated by the Hertzian dipole and the secondary field due to the induced electric current on the surface of the cube. Coefficients for the discretized induced current are provided by the iterative algorithm at each iteration. Theoretically, the total electric field should be zero outside the cube, because the source is shielded by a perfectly-conducting closed surface. Therefore, we define the "far-field error" as

$$
e_{f}=\frac{\left\|E_{t o t}^{\infty}\right\|_{2}}{\left\|\boldsymbol{E}_{\text {inc }}^{\infty}\right\|_{2}}
$$

where $\boldsymbol{E}_{t o t}^{\infty}$ and $\boldsymbol{E}_{\text {inc }}^{\infty}$ are arrays of 360 elements containing total and incident electric fields in the far zone.

In the second row of Fig. 4, the far-field error (22) in the EFIE and CFIE solutions is plotted with respect to iterations. We observe that the far-field error in the EFIE solution is reduced to $2 \times 10^{-3}$ at the 150th iteration, but it becomes steady and does not drop after this point, even though the residual error continues to decrease. Our observations for the far-field error in the CFIE solutions can be listed as follows.

- Similar to the EFIE solution, accuracy of the CFIE solutions is saturated and cannot be improved any further after some numbers of iterations.

- The last useful iteration and the minimum achievable accuracy in a CFIE solution depends on the value of $\alpha$.

- For each value of $\alpha$, the last useful iteration in terms of the solution accuracy corresponds to the breakpoint, where the compatibility of the CFIE solution with the EFIE system is saturated.

As a result, the compatibility of the CFIE solution with the EFIE system is an important indicator in determining the last useful iteration for the highest possible accuracy with CFIE.

Fig. 5 presents the iterative solutions of the scattering problem in Fig. 1(b). Similar to the previous example, the residual error is plotted as a function of BiCGStab iterations for EFIE and CFIE with $\alpha=0.2,0.5$, and 0.8. In the first row of Fig. 5, we again plot $\epsilon_{C \rightarrow E}$ and $\epsilon_{C \rightarrow M}$ to demonstrate the compatibility of the CFIE solutions with the EFIE and MFIE systems. For this problem, saturation occurs at the 7th, 

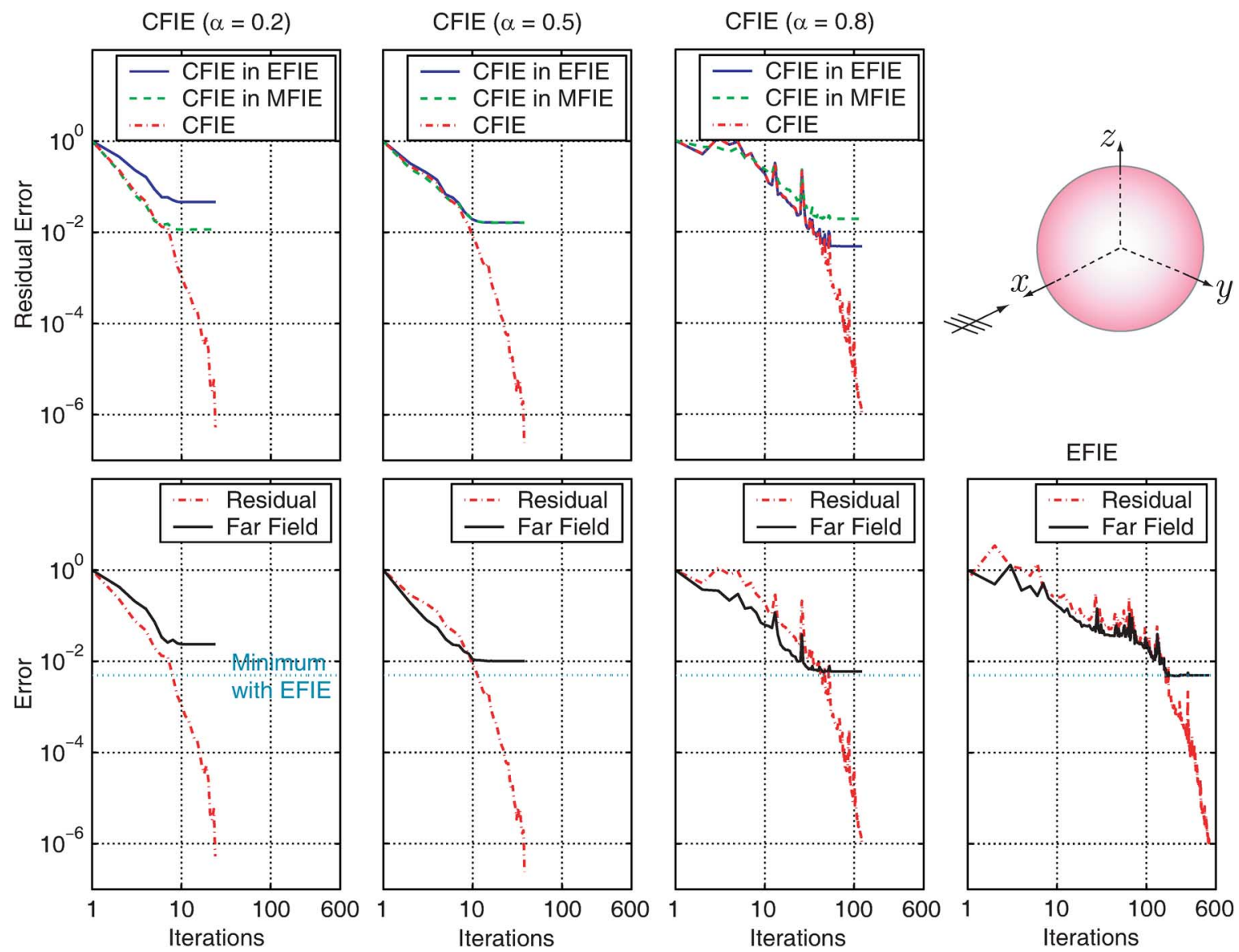

Fig. 6. Iterative solutions of the scattering problem in Fig. 1(c). Residual errors and the far-field error defined in (25) are plotted as a function BiCGStab iterations for EFIE and CFIE when $\alpha=0.2,0.5$, and 0.8 .

10th, and 30th iterations, respectively, when $\alpha=0.2,0.5$, and 0.8 . During the solutions, we also calculate the error in the near-zone electric field at each iteration. In this case, we sample the total electric field inside the cube at $19 \times 19=361$ regularly-spaced points, i.e.,

$$
\begin{aligned}
E_{t o t}[p]=\left\{\left|E_{\text {tot }}^{x}\left(x_{p}, y_{p}, z\right)\right|^{2}\right. & +\left|E_{\text {tot }}^{y}\left(x_{p}, y_{p}, z\right)\right|^{2} \\
& \left.+\left|E_{\text {tot }}^{z}\left(x_{p}, y_{p}, z\right)\right|^{2}\right\}^{1 / 2}
\end{aligned}
$$

where $E_{\text {tot }}^{x}, E_{t o t}^{y}$, and $E_{t o t}^{z}$ are the $x, y$, and $z$ components of the total electric field, $z=0$, and $-\lambda / 2 \leq x_{p}, y_{p} \leq \lambda / 2$ for $p=1,2, \ldots, 361$. Because of the shielding effect of the perfectly-conducting closed surface, the total electric field, which is obtained by combining the incident plane wave and the secondary field due to the induced electric current, should be zero inside the cube. Then, we define the "near-field error" as

$$
e_{n}=\frac{\left\|\boldsymbol{E}_{t o t}\right\|_{2}}{\left\|\boldsymbol{E}_{\text {inc }}\right\|_{2}}
$$

where $\boldsymbol{E}_{t o t}$ and $\boldsymbol{E}_{i n c}$ are arrays of 361 elements containing total and incident electric fields at the sample points. As depicted in the second row of Fig. 5, the near-field error in the EFIE solution is saturated at the 150th iteration, and the minimum achievable error is approximately $2.9 \times 10^{-3}$. In addition, similar to the previous example, the last useful iteration for the minimum possible error with CFIE coincides with the breakpoint for the compatibility of the solution with the EFIE system.

Finally, Fig. 6 presents the iterative solutions of the problem of scattering from a sphere, as illustrated in Fig. 1(c). This problem can be solved analytically using the Mie-series formalism. As depicted in the first row of Fig. 6, the compatibility of the CFIE solutions with the EFIE system saturates at the 9th, 13th, and 56th iterations, respectively, when $\alpha=0.2,0.5$, and 0.8 . During the iterative solutions of this problem, "the far-field error" is calculated as

$$
e_{f}=\frac{\left\|E_{a-c}^{\infty}\right\|_{2}}{\left\|\boldsymbol{E}_{a}^{\infty}\right\|_{2}}
$$

where

$$
\begin{aligned}
& E_{a}^{\infty}[p]=\lim _{R \rightarrow \infty} R\left\{\left|E_{a}^{\theta}\left(R, \theta, \phi_{p}\right)\right|^{2}+\left|E_{a}^{\phi}\left(R, \theta, \phi_{p}\right)\right|^{2}\right\}^{1 / 2} \\
& E_{a-c}^{\infty}[p]=\lim _{R \rightarrow \infty} R\left\{\left|E_{a}^{\theta}\left(R, \theta, \phi_{p}\right)-E_{c}^{\theta}\left(R, \theta, \phi_{p}\right)\right|^{2}\right. \\
&\left.+\left|E_{a}^{\phi}\left(R, \theta, \phi_{p}\right)-E_{c}^{\phi}\left(R, \theta, \phi_{p}\right)\right|^{2}\right\}^{1 / 2}
\end{aligned}
$$

$\theta=\pi / 2$, and $\phi_{p}=(p-1) \pi / 180$ for $p=1,2, \ldots, 360$. In (26) and (27), $E_{a}^{\theta}$ and $E_{a}^{\phi}$ are the $\theta$ and $\phi$ components of the electric field obtained by a reference Mie series solution, whereas 
$E_{c}^{\theta}$ and $E_{c}^{\phi}$ represent computational values. Fig. 6 confirms the previous results that the minimum achievable error and the last useful iteration in the CFIE solutions are closely related to the compatibility of the solutions with the EFIE system.

\section{CONCLUding REMARKS}

In this study, we present our investigations on the accuracy of CFIE discretized with the RWG functions. Our observations can be summarized as follows.

- Due to the excessive discretization error of the identity operator in MFIE [21], matrix equations obtained with EFIE and MFIE are incompatible. Being a convex combination of EFIE and MFIE, CFIE is contaminated with the MFIE inaccuracy, and CFIE solutions are also incompatible with the EFIE and MFIE systems.

- In an iterative solution of a matrix equation obtained from the discretization of an integral-equation formulation, accuracy of the solution is saturated and the solution error cannot be reduced any further after a number of iterations. This is because there are various error sources, and the overall error cannot be minimized by only reducing the residual error.

- Minimization of the residual vector during an iterative solution of CFIE involves a breakpoint, where the compatibility of the solution with the EFIE and MFIE systems cannot be enhanced any further.

- Since EFIE is more accurate than CFIE, the breakpoint for the compatibility of the CFIE solution with the EFIE system also corresponds to the last useful iteration, where the minimum possible error is achieved with CFIE.

We conclude that the accuracy of CFIE solutions are contaminated with the well-known inaccuracy of MFIE, that the accuracy of CFIE solutions cannot be improved simply by reducing the residual error in an iterative solution, and that the compatibility of the CFIE solution with the EFIE system is an important indicator to determine the last useful iteration to obtain the highest possible accuracy with CFIE. Even though these conclusions are deduced from the results of radiation and scattering problems involving canonical objects in this study, the same conclusions are also confirmed with various other problems involving conducting objects with diverse geometries.

\section{REFERENCES}

[1] A. J. Poggio and E. K. Miller, "Integral equation solutions of three-dimensional scattering problems," in Computer Techniques for Electromagnetics, R. Mittra, Ed. Oxford, U.K.: Pergamon Press, 1973, ch. 4.

[2] J. R. Mautz and R. F. Harrington, "H-field, E-field, and combined field solutions for conducting bodies of revolution," $A E \ddot{U}$, vol. 32, no. 4, pp. 157-164, Apr. 1978.

[3] D. R. Wilton and J. E. Wheeler, III, "Comparison of convergence rates of the conjugate gradient method applied to various integral equation formulations," Prog. Electromagn. Res., pp. 131-158, 1991.

[4] L. Gürel and Ö. Ergül, "Comparisons of FMM implementations employing different formulations and iterative solvers," in Proc. IEEE Antennas and Propag. Soc. Int. Symp., 2003, vol. 1, pp. 19-22.

[5] L. Gürel and Ö. Ergül, "Extending the applicability of the combinedfield integral equation to geometries containing open surfaces," IEEE Antennas Wireless Propag. Lett., vol. 5, pp. 515-516, 2006.
[6] R. Coifman, V. Rokhlin, and S. Wandzura, "The fast multipole method for the wave equation: A pedestrian prescription," IEEE Antennas Propag. Mag., vol. 35, no. 3, pp. 7-12, Jun. 1993.

[7] J. Song, C.-C. Lu, and W. C. Chew, "Multilevel fast multipole algorithm for electromagnetic scattering by large complex objects," IEEE Trans. Antennas Propag., vol. 45, no. 10, pp. 1488-1493, Oct. 1997.

[8] S. M. Rao, D. R. Wilton, and A. W. Glisson, "Electromagnetic scattering by surfaces of arbitrary shape," IEEE Trans. Antennas Propag., vol. 30, no. 3, pp. 409-418, May 1982.

[9] Ö. Ergül and L. Gürel, "Investigation of the inaccuracy of the MFIE discretized with the RWG basis functions," in Proc. IEEE Antennas and Propag. Soc. Int. Symp., 2004, vol. 3, pp. 3393-3396.

[10] Ö. Ergül and L. Gürel, "Improving the accuracy of the MFIE with the choice of basis functions," in Proc. IEEE Antennas and Propag. Soc. Int. Symp., 2004, vol. 3, pp. 3389-3392.

[11] C. P. Davis and K. F. Warnick, "High-order convergence with a low-order discretization of the 2-D MFIE," IEEE Antennas Wireless Propag. Lett., vol. 3, pp. 355-358, 2004.

[12] E. Ubeda and J. M. Rius, "MFIE MOM-formulation with curl-conforming basis functions and accurate kernel integration in the analysis of perfectly conducting sharp-edged objects," Microw. Opt. Technol. Lett., vol. 44, no. 4, pp. 354-358, Feb. 2005.

[13] E. Ubeda and J. M. Rius, "Monopolar divergence-conforming and curl-conforming low-order basis functions for the electromagnetic scattering analysis," Microw. Opt. Technol. Lett., vol. 46, no. 3, pp. 237-241, Aug. 2005.

[14] E. Ubeda and J. M. Rius, "Novel monopolar MFIE MoM-discretization for the scattering analysis of small objects," IEEE Trans. Antennas Propag., vol. 54, no. 1, pp. 50-57, Jan. 2006.

[15] Ö. Ergül and L. Gürel, "The use of curl-conforming basis functions for the magnetic-field integral equation," IEEE Trans. Antennas Propag., vol. 54, no. 7, pp. 1917-1926, Jul. 2006.

[16] Ö. Ergül and L. Gürel, "Improving the accuracy of the magnetic field integral equation with the linear-linear basis functions," Radio Sci., vol. 41, p. RS4004, Jul. 2006, 10.1029/2005RS003307.

[17] Ö. Ergül and L. Gürel, "On the accuracy of MFIE and CFIE in the solution of large electromagnetic scattering problems," presented at the Eur. Conf. on Antennas and Propag. (EuCAP), 2006.

[18] Ö. Ergül and L. Gürel, "Linear-linear basis functions for MLFMA solutions of magnetic-field and combined-field integral equations," IEEE Trans. Antennas Propag., vol. 55, no. 4, pp. 1103-1110, Apr. 2007.

[19] K. F. Warnick and A. F. Peterson, "3D MFIE accuracy improvement using regularization," in Proc. IEEE Antennas and Propag. Soc. Int. Symp., 2007, pp. 4857-4860.

[20] P. Ylä-Oijala, M. Taskinen, and S. Järvenpää, "Analysis of surface integral equations in electromagnetic scattering and radiation problems," Eng. Anal. Boundary Elem., vol. 32, no. 3, pp. 196-209, Mar. 2008.

[21] Ö. Ergül and L. Gürel, "Discretization error due to the identity operator in surface integral equations," Comp. Phys. Comm., to be published.

[22] R. D. Graglia, "On the numerical integration of the linear shape functions times the 3-D Green's function or its gradient on a plane triangle," IEEE Trans. Antennas Propag., vol. 41, no. 10, pp. 1448-1455, Oct. 1993.

[23] R. E. Hodges and Y. Rahmat-Samii, "The evaluation of MFIE integrals with the use of vector triangle basis functions," Microw. Opt. Technol. Lett., vol. 14, no. 1, pp. 9-14, Jan. 1997.

[24] P. Ylä-Oijala and M. Taskinen, "Calculation of CFIE impedance matrix elements with RWG and $\hat{\boldsymbol{n}} \times$ RWG functions," IEEE Trans. Antennas Propag., vol. 51, no. 8, pp. 1837-1846, Aug. 2003.

[25] L. Gürel and Ö. Ergül, "Singularity of the magnetic-field integral equation and its extraction," IEEE Antennas Wireless Propag. Lett., vol. 4, pp. 229-232, 2005.

[26] D. A. Dunavant, "High degree efficient symmetrical Gaussian quadrature rules for the triangle," Int. J. Numer. Meth. Eng., vol. 21, pp. 1129-1148, 1985.

[27] Ö. S. Ergül, "Fast Multipole Method for the Solution of Electromagnetic Scattering Problems," M.S. thesis, Bilkent Univ., Ankara, Turkey, Jun. 2003.

[28] H. van der Vorst, "Bi-CGSTAB: A fast and smoothly converging variant of Bi-CG for the solution of nonsymmetric linear systems," SIAM J. Sci. Stat. Comput., vol. 13, no. 2, pp. 631-644, Mar. 1992. 


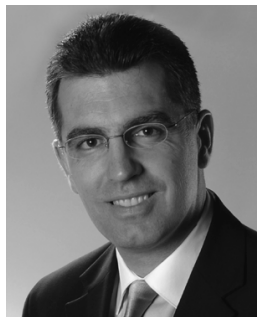

Levent Gürel (S'87-M'92-SM'97-F'09) received the B.Sc. degree from the Middle East Technical University (METU), Ankara, Turkey, in 1986, and the M.S. and Ph.D. degrees from the University of Illinois at Urbana-Champaign (UIUC) in 1988 and 1991, respectively, all in electrical engineering.

He joined the Thomas J. Watson Research Center of the International Business Machines Corporation, Yorktown Heights, New York, in 1991, where he worked as a Research Staff Member on the electromagnetic compatibility (EMC) problems related to electronic packaging, on the use of microwave processes in the manufacturing and testing of electronic circuits, and on the development of fast solvers for interconnect modeling. Since 1994, he has been a faculty member in the Department of Electrical and Electronics Engineering of the Bilkent University, Ankara, where he is currently a Professor. He was a Visiting Associate Professor at the Center for Computational Electromagnetics (CCEM) of the UIUC for one semester in 1997. He returned to the UIUC as a Visiting Professor in 2003-2005, and as an Adjunct Professor after 2005. He founded the Computational Electromagnetics Research Center (BiLCEM) at Bilkent University in 2005, where he is serving as the Director. Prof. Gürel's research interests include the development of fast algorithms for computational electromagnetics (CEM) and the application thereof to scattering and radiation problems involving large and complicated scatterers, antennas and radars, frequency-selective surfaces, high-speed electronic circuits, optical and imaging systems, nanostructures, and metamaterials. He is also interested in the theoretical and computational aspects of electromagnetic compatibility and interference analyses. Ground penetrating radars and other subsurface scattering applications are also among his research interests. Since 2006, his research group has been breaking several world records by solving extremely large integral-equation problems, most recently the largest involving as many as 205 million unknowns.

Prof. Gürel's many awards for his accomplishments include the Turkish Academy of Sciences (TUBA) in 2002 and the Scientific and Technical Research Council of Turkey (TUBITAK) in 2003, as the most notable. He served as the Chairman of the AP/MTT/ED/EMC Chapter of the IEEE Turkey Section from 2000 to 2003. He founded the IEEE EMC Chapter in Turkey in 2000.
He served as the Co-chairman of the 2003 IEEE International Symposium on Electromagnetic Compatibility. He is the organizer and General Chair of the CEM'07 and CEM'09 Computational Electromagnetics International Workshops held in 2007 and 2009. He is a member of the USNC of the International Union of Radio Science (URSI) and the Chairman of Commission E (Electromagnetic Noise and Interference) of URSI Turkey National Committee. He served as a member of the General Assembly of the European Microwave Association (EuMA) during 2006-2008. He is currently serving as an Associate Editor for the IEEE ANTENNAS AND WIRELESS PROPAGATION LETTERS, Radio Science, Journal of Electromagnetic Waves and Applications (JEMWA), and Progress in Electromagnetics Research (PIER).

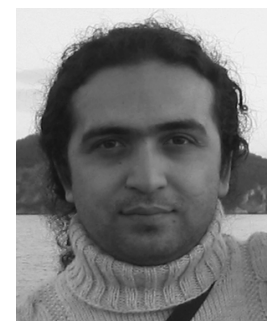

Özgür Ergül (S'98) received B.Sc. and M.S. degrees in electrical and electronics engineering from Bilkent University, Ankara, Turkey, in 2001 and 2003 , respectively. He is currently working toward the $\mathrm{Ph} . \mathrm{D}$. degree at Bilkent University.

Since 2001, he has served as a Teaching and Research Assistant in the Department of Electrical and Electronics Engineering, Bilkent University. $\mathrm{He}$ has been affiliated with the Computational Electromagnetics Group at Bilkent University from 2000 to 2005 and with the Computational Electromagnetics Research Center (BiLCEM) since 2005. His research interests include fast and accurate algorithms for the solution of electromagnetics problems involving large and complicated structures, integral equations, parallel programming, and iterative techniques.

Mr. Ergül is a recipient of the 2007 IEEE Antennas and Propagation Society Graduate Fellowship and the 2007 Leopold B. Felsen Award for Excellence in Electrodynamics. He is the Secretary of Commission E (Electromagnetic Noise and Interference) of URSI Turkey National Committee. His academic endeavors are supported by the Scientific and Technical Research Council of Turkey (TUBITAK) through a Ph.D. scholarship. 